\title{
Exactly Solvable Magnet of Conformal Spins in Four Dimensions
}

\author{
Sergey Derkachov $\oplus^{1}$ and Enrico Olivucci $\odot^{2}$ \\ ${ }^{1}$ St. Petersburg Department of the Steklov Mathematical Institute, St. Petersburg 191023, Russia \\ ${ }^{2}$ II. Institute for Theoretical Physics of the University of Hamburg, Hamburg 22761, Germany
}

(Received 13 April 2020; accepted 23 June 2020; published 17 July 2020)

\begin{abstract}
We provide the eigenfunctions for a quantum chain of $N$ conformal spins with nearest-neighbor interaction and open boundary conditions in the irreducible representation of $\mathrm{SO}(1,5)$ of scaling dimension $\Delta=2-i \lambda$ and spin numbers $\ell=\dot{\ell}=0$. The spectrum of the model is separated into $N$ equal contributions, each dependent on a quantum number $Y_{a}=\left[\nu_{a}, n_{a}\right]$ which labels a representation of the principal series. The eigenfunctions are orthogonal and we computed the spectral measure by means of a new star-triangle identity. Any portion of a conformal Feynmann diagram with square lattice topology can be represented in terms of separated variables, and we reproduce the all-loop "fishnet" integrals computed by B. Basso and L. Dixon via bootstrap techniques. We conjecture that the proposed eigenfunctions form a complete set and provide a tool for the direct computation of conformal data in the fishnet limit of the supersymmetric $\mathcal{N}=4$ Yang-Mills theory at finite order in the coupling, by means of a cutting-and-gluing procedure on the square lattice.
\end{abstract}

DOI: $10.1103 /$ PhysRevLett.125.031603

Introduction.-The exactly solvable spin magnets $[1,2]$ constitute a class of condensed matter models of wide interest throughout theoretical and mathematical physics. In particular, the integrable chains of nearest-neighbors interacting spins [3,4] serve as a tool to encode the symmetries of local or nonlocal operators in quantum field theory, providing a rich amount of nonperturbative results ranging from the scattering spectrum of high-energy gluons in QCD [5-7] to the conformal data of the $\mathcal{N}=4$ supersymmetric Yang-Mills and $\mathcal{N}=6$ Aharony-BergmanJafferis-Maldacena theories [8]. The archetype model of this class is the $\mathrm{SU}(2)$ Heisenberg magnet of spin $\frac{1}{2}$, which for open boundary conditions is described by the Hamiltonian

$$
H_{\mathrm{SU}(2)}=\sum_{a=1}^{N-1} \vec{\sigma}_{a} \vec{\sigma}_{a+1},
$$

being $\vec{\sigma}_{a}$ the vector of Pauli matrices acting on the space $\mathbb{V}_{a}=\mathbb{C}^{2}$. Generalizations of (1) to other symmetry groups are known, including the noncompact $\mathrm{SO}(1,5)$ spin chain [9]. The latter model is relevant for the study of covariant quantities in a four-dimensional conformal field theory (CFT) [10]. We consider the homogeneous model in the irreducible unitary representation defined by the scaling

Published by the American Physical Society under the terms of the Creative Commons Attribution 4.0 International license. Further distribution of this work must maintain attribution to the author(s) and the published article's title, journal citation, and DOI. Funded by SCOAP ${ }^{3}$. dimension $\Delta=2-i \lambda, \lambda \in \mathbb{R}$, and the $\mathrm{SO}(4)$ spins $\ell=$ $\dot{\ell}=0$ [11]. The Hamiltonian operator acts on the Hilbert spaces $\mathbb{V}_{a}=L^{2}\left(x_{a}, d^{4} x_{a}\right)$ as

$$
\begin{aligned}
\mathbb{U}= & \sum_{a=1}^{N-1}\left[2 \ln x_{a a+1}^{2}+\left(x_{a a+1}^{2}\right)^{-i \lambda} \ln \left(\hat{p}_{a}^{2} \hat{p}_{a+1}^{2}\right)\left(x_{a a+1}^{2}\right)^{i \lambda}\right] \\
& +2 \ln x_{N 0}^{2}+\ln \left(\hat{p}_{1}^{2}\right)+\left(x_{N 0}^{2}\right)^{-i \lambda} \ln \left(p_{N}^{2}\right)\left(x_{N 0}^{2}\right)^{i \lambda}
\end{aligned}
$$

where $x_{a a+1}=x_{a}-x_{a+1}, \hat{p}_{a}^{2}=-\partial_{a} \partial_{a}$ and $x_{N+1}=x_{0}$. The point $x_{0}$ is effectively a parameter for the model, and we will always omit it from the set of coordinates. The spin chain (2) is the four-dimensional version of the open $S L(2, \mathbb{C})$ Heisenberg magnet which describes the scattering amplitudes of high energy gluons in the Regge limit of QCD [7,12]. The integrability of (2) is realized by the commutative family of normal operators

$$
\mathbb{Q}_{N}(u)=Q_{12}(u) Q_{23}(u) \cdots Q_{N 0}(u),
$$

labeled by the spectral parameter $u \in \mathbb{R}$ and where

$$
Q_{i j}(u)=\left(x_{i j}^{2}\right)^{-i \lambda}\left(\hat{p}_{i}^{2}\right)^{u}\left(x_{i j}^{2}\right)^{u+i \lambda} .
$$

By the introduction of the operator

$$
\hat{\mathbb{Q}}_{N}(u)=\left[\mathbb{Q}_{N}(u-i \lambda)\right]^{\dagger} \mathbb{Q}_{N}(-i \lambda),
$$

the Hamiltonian $\mathbb{W}$ is recovered from the expansion

$$
\mathbb{Q}_{N}(u)+\hat{\mathbb{Q}}_{N}(u)=2 \mathbb{1}+u \llbracket+o(u) .
$$


It follows from (4) and from the commutation relation $\left[\mathbb{Q}_{N}(u), \mathbb{Q}_{N}(v)\right]=0$ at generic $u$ and $v$, that the eigenfunctions of $\mathbb{Q}_{N}$ diagonalize the Hamiltonian (2) as well. The spectra of these operators are labeled by the quantum numbers

$$
\begin{aligned}
& Y_{a}=1+\frac{n_{a}}{2}+i \nu_{a}, \quad Y_{a}^{*}=1+\frac{n_{a}}{2}-i \nu_{a}, \\
& \nu_{a} \in \mathbb{R}, \quad n_{a} \in \mathbb{N},
\end{aligned}
$$

for $a=1, \ldots, N$, and we use to write $\mathbf{Y}=\left(Y_{1}, \ldots, Y_{N}\right)$. The spectral equation for the operator (3) reads

$$
\mathbb{Q}_{N}(u) \Psi^{\alpha \beta}(\mathbf{x} \mid \mathbf{Y})=\tau_{N}(u, \mathbf{Y}) \Psi^{\alpha \beta}(\mathbf{x} \mid \mathbf{Y}),
$$

where we denote $\mathbf{x}=\left(x_{1}, \ldots, x_{N}\right)$ and $\boldsymbol{\alpha}, \boldsymbol{\beta}$ stand for $2 N$ auxiliary complex spinors

$$
\left|\alpha_{1}\right\rangle, \ldots,\left|\alpha_{N}\right\rangle \quad \text { and } \quad\left|\beta_{1}\right\rangle, \ldots,\left|\beta_{N}\right\rangle \in \mathbb{C}^{2} .
$$

The eigenfunctions form an orthogonal set respect to the quantum numbers $(\mathbf{Y}, \boldsymbol{\alpha}, \boldsymbol{\beta})$, and the eigenvalue is factorized respect to the labels (5) into equal contributions

$$
\begin{aligned}
\tau_{N}(u, \mathbf{Y}) & =\prod_{a=1}^{N} \tau_{1}\left(u, Y_{a}\right), \\
\tau_{1}\left(u, Y_{a}\right) & =4^{u} \frac{\Gamma\left(Y_{a}-\frac{i}{2} \lambda\right) \Gamma\left(Y_{a}^{*}+u+\frac{i}{2} \lambda\right)}{\Gamma\left(Y_{a}^{*}+\frac{i}{2} \lambda\right) \Gamma\left(Y_{a}-u-\frac{i}{2} \lambda\right)} .
\end{aligned}
$$

As a consequence of (4) and (6) we obtained the spectrum of the Hamiltonian $\mathbb{H}$ as a sum of $N$ independent terms

$\eta_{N}(\mathbf{Y})=\sum_{a=1}^{N}\left[\psi\left(Y_{a}-\frac{i}{2} \lambda\right)+\psi\left(Y_{a}+\frac{i}{2} \lambda\right)+\ln 4\right]+$ c.c.

Formulas (6), (7) show that the $N$-body system defined in (2) gets separated into $N$ one-particle systems over the quantum numbers (5). In other words, the quantities $\left(Y_{a},\left|\alpha_{a}\right\rangle,\left|\beta_{a}\right\rangle\right)$ are the separated variables of the system in the sense of [13-16], and the spectrum of (2) and (3) is degenerate in the spinors due to rotation invariance.

The representation over the separated variables $(\mathbf{Y}, \boldsymbol{\alpha}, \boldsymbol{\beta})$ is defined for a generic function $\phi(\mathbf{x})=\phi\left(x_{1}, \ldots, x_{N}\right)$ by the linear transform

$$
\tilde{\phi}(\mathbf{Y}, \boldsymbol{\alpha}, \boldsymbol{\beta})=\int d \mathbf{x} \Psi^{\alpha \beta}(\mathbf{x} \mid \mathbf{Y})^{*} \phi(\mathbf{x}) .
$$

The inverse transform of (8) provides the expansion of $\phi(\mathbf{x})$ over the basis of eigenfunctions $\phi(\mathbf{x})=\sum_{\mathbf{n}} \int d \boldsymbol{\nu} \mu(\mathbf{Y}) \int D \alpha D \boldsymbol{\beta} \Psi^{\alpha \beta}(\mathbf{x} \mid \mathbf{Y}) \tilde{\phi}(\mathbf{Y}, \boldsymbol{\alpha}, \boldsymbol{\beta})$,

where the sum runs over the non-negative integers $\mathbf{n}=\left(n_{1}, \ldots, n_{N}\right)$, the integrations $d \boldsymbol{\nu}=d \nu_{1} \cdots d \nu_{N}$ are on the real line and the integration in the space of spinors $D \alpha=D \alpha_{1} \cdots D \alpha_{N}$ is defined as

$$
\int D \alpha=\int_{\mathbb{C}^{2}} d \alpha e^{-\langle\alpha \mid \alpha\rangle}, \quad\langle\alpha \mid \alpha\rangle=\left|\alpha^{(1)}\right|^{2}+\left|\alpha^{(2)}\right|^{2} .
$$

The spectral measure in (9) can be extracted from the scalar product of eigenfunctions and it is given by

$$
\begin{aligned}
\mu(\mathbf{Y})= & \frac{1}{N !} \prod_{a=1}^{N}\left(n_{a}+1\right) \prod_{b \neq a}^{N}\left[\nu_{a b}^{2}+\frac{n_{a b}^{2}}{4}\right] \\
& \times\left[\nu_{a b}^{2}+\frac{\left(n_{a}+n_{b}+2\right)^{2}}{4}\right]
\end{aligned}
$$

in the notation $\nu_{a b}=\nu_{a}-\nu_{b}$ and $n_{a b}=n_{a}-n_{b}$.

All considerations done so far can be extended by an accurate analytic continuation of the parameter $\lambda$ to the imaginary strip $(-2 i,+2 i)$. In particular, at $\lambda=-i$ each site of the chain carries the representation $\Delta=1, \ell=\dot{\ell}=$ 0 of a bare scalar field in four dimensions. In this case at the point $u=-1$ the operator $\mathbb{Q}_{N}(u)$ becomes proportional to the graph-building integral operator for a Feynmann diagram of square lattice topology

$$
\mathbb{B}_{N} \phi(\mathbf{x})=\frac{1}{(2 \pi)^{4 N}} \int d \mathbf{x}^{\prime} \phi\left(\mathbf{x}^{\prime}\right) \prod_{a=1}^{N} \frac{1}{x_{a a+1}^{2} x_{a a^{\prime}}^{2}},
$$

with $\mathbf{x}=\left(x_{1}, \ldots, x_{N}\right), \mathbf{x}^{\prime}=\left(x_{1}^{\prime}, \ldots, x_{N}^{\prime}\right)$. Throughout the Letter we denote $x_{a b^{\prime}}=x_{a}-x_{b}^{\prime}$. According to (6) the representation of the operator $\left(\mathbb{B}_{N}\right)^{L}$ over the separated variables factorizes completely a portion of size $N \times L$ of the planar fishnet diagram [17] in Fig. 1, which extends to a 4D space-time the analog result in two-dimensions of [18]. As a direct application of our results, we computed a specific set of four-point functions of Fishnet CFT [19], providing a direct check to formula (14) of [20], obtained via arguments of AdS/CFT correspondence [21-23]. In the next two sections we present the explicit construction of the eigenfunctions of the model (2) by means of newly found integral identities.

Generalized star-triangle identity.-Our construction of a basis of eigenfunctions for $\mathbb{Q}_{N}(u)$ follows the logic outlined in [24] for the two-dimensional model, and requires the formulation of certain conformal integral identities in 4D.

First of all we consider two conformal propagators defined by the scaling dimensions $a$ and $b$, and transforming under rotations $\mathrm{SO}(4)$ in the symmetric traceless tensor representations of rank $n$ and $n^{\prime} \in \mathbb{N}$ 

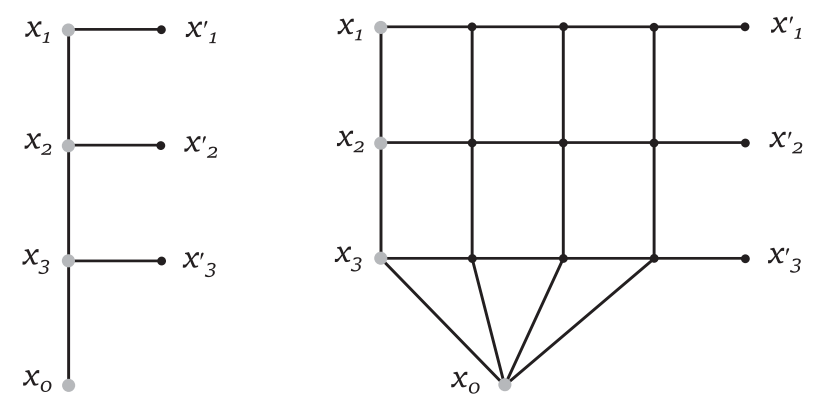

FIG. 1. On the left the graph-building kernel $\mathbb{B}_{3}\left(\mathbf{x} \mid \mathbf{x}^{\prime}\right)$, where the lines are propagators $1 / x_{i j}^{2}$, grey dots are external points and the black ones are integrated. On the right the portion of fishnet $\left(\mathbb{B}_{3}\right)^{4}$ with two fixed points $x_{0}$ (down) and $\infty$ (up).

$$
\frac{\langle\alpha|\overline{\mathbf{x}}| \beta\rangle^{n}}{\left(x^{2}\right)^{a}}, \quad \frac{\left\langle\alpha^{\prime}|\mathbf{x}| \beta^{\prime}\right\rangle^{n^{\prime}}}{\left(x^{2}\right)^{b}}
$$

where the tensor indices are contracted with a null vector as $\langle\alpha|\overline{\mathbf{x}}| \beta\rangle=\left\langle\alpha\left|\overline{\boldsymbol{\sigma}}^{\mu}\right| \beta\right\rangle x_{\mu} /|x|$, and the symbols $\boldsymbol{\sigma}$ and $\overline{\boldsymbol{\sigma}}$ are defined in terms of Pauli matrices

$$
\boldsymbol{\sigma}_{0}=\overline{\boldsymbol{\sigma}}_{0}=\mathbb{1}, \quad \boldsymbol{\sigma}_{k}=-\overline{\boldsymbol{\sigma}}_{k}=i \sigma_{k}, \quad k=1,2,3 .
$$

The convolution of the two propagators (12) is given by the integral identity

$$
\begin{aligned}
\int d^{4} y \frac{\langle\alpha|\overline{\mathbf{y}}| \beta\rangle^{n}}{\left(y^{2}\right)^{a}} \frac{\left\langle\alpha^{\prime}|\mathbf{y}-x| \beta^{\prime}\right\rangle^{n^{\prime}}}{\left[(x-y)^{2}\right]^{b}}=\pi^{2} \frac{A_{n, n^{\prime}}(a, b)}{\left(x^{2}\right)^{(a+b-2)}} \\
\times \frac{\left\langle\alpha, \alpha^{\prime} \mathbf{x}\left|\mathbf{R}_{n, n^{\prime}}(2-a-b)\right| \overline{\mathbf{x}} \beta, \beta^{\prime}\right\rangle}{\left(3-a-b+\frac{n+n^{\prime}}{2}\right)},
\end{aligned}
$$

with the coefficient $A_{n, n^{\prime}}(a, b)=$

$$
=\frac{\Gamma\left(2-a+\frac{n}{2}\right) \Gamma\left(2-b+\frac{n^{\prime}}{2}\right) \Gamma\left(a+b-2+\frac{n^{\prime}-n}{2}\right)}{\Gamma\left(a+\frac{n}{2}\right) \Gamma\left(b+\frac{n^{\prime}}{2}\right) \Gamma\left(3-a-b+\frac{n^{\prime}-n}{2}\right)},
$$

and $\mathbf{R}_{n, n^{\prime}}(z)$ is a compact expression for the mixing of symmetric spinors $\left|\beta, \beta^{\prime}\right\rangle=|\beta\rangle^{\otimes n} \otimes\left|\beta^{\prime}\right\rangle{ }^{\otimes n^{\prime}}$ produced by the integration, and defined by the matrix element

$$
\begin{aligned}
& \left\langle\alpha, \alpha^{\prime}\left|\mathbf{R}_{n, n^{\prime}}(z)\right| \beta, \beta^{\prime}\right\rangle=\frac{\Gamma\left(z+1+\frac{n-n^{\prime}}{2}\right) \Gamma\left(z+1+\frac{n^{\prime}-n}{2}\right)}{\Gamma\left(z+1+\frac{n+n^{\prime}}{2}\right)^{2}} \\
& \times \frac{d^{n}}{d s^{n}} \frac{d^{n^{\prime}}}{d k^{n^{\prime}}}\left(1+s\langle\alpha \mid \beta\rangle+k\left\langle\alpha^{\prime} \mid \beta^{\prime}\right\rangle+s k\left\langle\alpha \mid \beta^{\prime}\right\rangle\left\langle\alpha^{\prime} \mid \beta\right\rangle\right)^{z+\left(n+n^{\prime}\right) / 2},
\end{aligned}
$$

where upon derivation we set the parameters $s, k=0$. We recognize that the matrix $\mathbf{R}_{n, n^{\prime}}(z)$-emerging from the convolution integral (13) - is a solution of the Yang-Baxter equation on the space of symmetric spinors
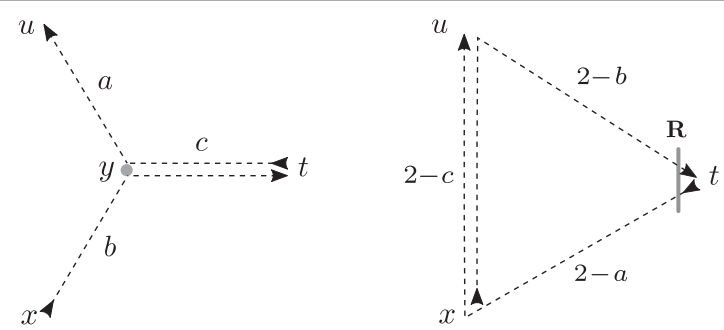

FIG. 2. Graphic representation of the identity (14). On the left, the star integral with the integration point $y$. The letter " $a$ " corresponds to the power of the inverse square distance $1 /\left[(u-y)^{2}\right]^{a}$, and similarly for the other lines. The arrows define the order of matrices $\boldsymbol{\sigma}$ and $\overline{\boldsymbol{\sigma}}$ as in the numerators of (14). On the right, the corresponding triangle, with the matrix $\mathbf{R}_{n, n^{\prime}}$ inserted as a grey line.

$$
\begin{aligned}
& \mathbf{R}_{n, n^{\prime}}(z-w) \mathbf{R}_{n, n^{\prime \prime}}(z) \mathbf{R}_{n^{\prime}, n^{\prime \prime}}(w) \\
= & \mathbf{R}_{n^{\prime}, n^{\prime \prime}}(w) \mathbf{R}_{n, n^{\prime \prime}}(z) \mathbf{R}_{n, n^{\prime}}(z-w),
\end{aligned}
$$

and can be obtained via the fusion procedure [25] applied to the Yangian $R$ matrix $\mathbf{R}_{1,1}(z)$.

The identity (13) is the core of the generalization of the uniqueness-"star-triangle"-relation for a conformal invariant vertex of three scalar propagators [26] (see also $[27,28]$ and references therein) to any symmetric traceless representation. Upon the chain of conformal transformations $x^{\mu} \rightarrow x^{\mu}-u^{\mu}, x^{\mu} \rightarrow x^{\mu} / x^{2}$, and $x^{\mu} \rightarrow x^{\mu}-t^{\mu}$, the convolution of propagators takes the form

$$
\begin{aligned}
& \int d^{4} y \frac{\langle\alpha|(\overline{\mathbf{t}-\mathbf{y}})(\mathbf{y}-\mathbf{u})| \beta\rangle^{n}\left\langle\alpha^{\prime}|(\overline{\mathbf{x}-\mathbf{y}})(\mathbf{y}-\mathbf{t})| \beta^{\prime}\right\rangle^{n^{\prime}}}{\left[(u-y)^{2}\right]^{a}\left[(x-y)^{2}\right]^{b}\left[(t-y)^{2}\right]^{c}}= \\
& =\frac{(-1)^{n}}{\left(c-1+\frac{n+n^{\prime}}{2}\right)}\left[\frac{\pi^{2} A_{n, n^{\prime}}(a, b)}{\left((x-t)^{2}\right]^{2-a}\left[(t-u)^{2}\right]^{2-b}\left[(u-x)^{2}\right]^{2-c}}\right. \\
& \times\left\langle\alpha, \alpha^{\prime}(\overline{\mathbf{x}-\mathbf{u}})(\mathbf{u}-\mathbf{t})\left|\mathbf{R}_{n, n^{\prime}}(c-2)\right|(\overline{\mathbf{t}-\mathbf{x}})(\mathbf{x}-\mathbf{u}) \beta, \beta^{\prime}\right\rangle,
\end{aligned}
$$

which holds under the uniqueness constraint $a+b+c=$ 4 and is the generalized star-triangle identity represented in Fig. 2. Setting $n^{\prime}=0$, the identity (14) is equivalent to (A.11) of [29], and setting further $n=0$ it degenerates to the scalar identity [26].

We point out that (14) is the four-dimensional version of the 2D star-triangle relation, which underlies the solution of the $S L(2, \mathbb{C})$ Heisenberg magnet as in $[24,30]$.

Eigenfunctions construction.-The eigenfunctions of the open conformal chain (2) can be obtained by a recursive procedure in the number of sites of the system. First we consider the model with only one site: at $N=1$ the eigenfunctions are fixed by conformal symmetry as propagators of scaling dimension $\Delta=1+i \lambda / 2+i \nu$ and tensor rank $n$ 

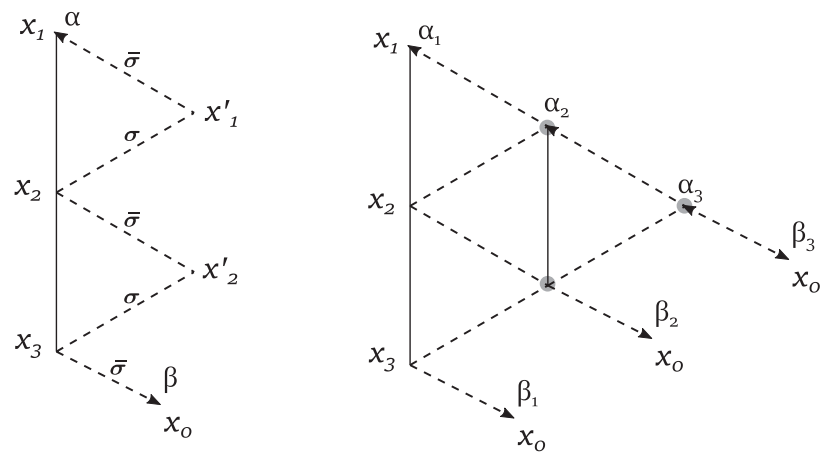

FIG. 3. Graphic representation of the integral kernel $\Lambda_{3, Y}^{\alpha, \beta}\left(x_{1}, x_{2}, x_{3} \mid x_{1}^{\prime}, x_{2}^{\prime}\right)$ (left) and of the eigenfunction $\Psi^{\alpha \alpha \beta}\left(\mathbf{Y} \mid x_{1}, x_{2}, x_{3}\right)$ (right). Solid lines denote $\left(x_{i j}^{2}\right)^{-i \lambda}$, while the dashed ones stand for the terms with matrices $\sigma, \bar{\sigma}$ in the numerator and carrying the variables $\nu$ in the power of the denominators. The external arrows indicate symmetric spinors and the grey blobs are integrated points.

$$
\Lambda_{1, Y}^{\alpha \beta}\left(x_{1}\right)=\frac{\left\langle\alpha\left|\overline{\mathbf{x}}_{10}\right| \beta\right\rangle^{n}}{\left(x_{10}^{2}\right)^{1+i \nu+i \lambda / 2}},
$$

and they solve the spectral problem

$$
\mathbb{Q}_{1}(u) \Lambda_{1, Y}^{\alpha \beta}\left(x_{1}\right)=\tau_{1}(u, Y) \Lambda_{1, Y}^{\alpha \beta}\left(x_{1}\right) .
$$

Let us denote $\mathbf{x}=\left(x_{1}, \ldots, x_{N}\right), \mathbf{x}^{\prime}=\left(x_{1}^{\prime}, \ldots, x_{N-1}^{\prime}\right)$. The direct generalization of (15) to the model with $N$ sites is given by the kernel function (see Fig. 3)

$$
\begin{aligned}
\Lambda_{N, Y}^{\alpha \beta}\left(\mathbf{x} \mid \mathbf{x}^{\prime}\right)= & \frac{\left\langle\alpha\left|\overline{\mathbf{x}}_{11^{\prime}} \mathbf{x}_{1^{\prime} 2} \overline{\mathbf{x}}_{22^{\prime}}, \ldots, \overline{\mathbf{x}}_{N 0}\right| \beta\right\rangle^{n}}{\left(x_{N 0}^{2}\right)^{1+i \nu+i \frac{\lambda}{2}}} \\
& \times \prod_{a=1}^{N-1} \frac{\left(x_{a^{\prime} a+1}^{2}\right)^{-1+i \nu+i(\lambda / 2)}}{\left(x_{a a^{\prime}}^{2}\right)^{1+i \nu-i(\lambda / 2)}\left(x_{a a+1}^{2}\right)^{i \lambda}} r(Y),
\end{aligned}
$$

where we introduced a suitable normalization

$$
r(Y)=\frac{\Gamma\left(Y-i \frac{\lambda}{2}\right) \Gamma\left(Y^{*}-i \frac{\lambda}{2}\right)}{\Gamma\left(Y+i \frac{\lambda}{2}\right) \Gamma\left(Y^{*}+i \frac{\lambda}{2}\right)}
$$

The building blocks of the eigenfunctions are the integral operators $\hat{\Lambda}_{M, Y}^{\alpha \beta}=\left\langle\alpha\left|\hat{\Lambda}_{M, Y}\right| \beta\right\rangle$, defined as

$$
\hat{\Lambda}_{M, Y}^{\alpha \beta} \phi(\mathbf{x})=\int d \mathbf{x}^{\prime} \Lambda_{M, Y}^{\alpha \beta}\left(\mathbf{x} \mid \mathbf{x}^{\prime}\right) \phi\left(\mathbf{x}^{\prime}\right) .
$$

Making use of (14) at $n=n_{a}, n^{\prime}=0$ we verify that

$$
\mathbb{Q}_{M}(u) \hat{\Lambda}_{M, Y}^{\alpha \beta}=\tau_{1}(u, Y) \hat{\Lambda}_{M, Y}^{\alpha \beta} \mathbb{Q}_{M-1}(u),
$$

for any $M>1$, therefore the iterative application of (18) for the length $M$ going from $N$ to 2 provides-together with the initial condition (16) - a recursive definition of the eigenfunctions of the model with $N$ sites

$$
\Psi^{\alpha \beta}(\mathbf{Y} \mid \mathbf{x})=\frac{1}{\left(2 \pi^{2 N+1}\right)^{\frac{N}{2}}} \hat{\Lambda}_{N, Y_{N}}^{\alpha_{N} \beta_{N}} \cdots \hat{\Lambda}_{2, Y_{2}}^{\alpha_{2} \beta_{2}} \Lambda_{1, Y_{1}}^{\alpha_{1} \beta_{1}} .
$$

The functions (19) show a simple behavior in the permutation of two separated variables $(Y, \alpha, \beta),\left(Y^{\prime}, \alpha^{\prime}, \beta^{\prime}\right)$, based on (14) and encoded by the exchange property

$$
\begin{array}{r}
\hat{\Lambda}_{M, Y^{\prime}}^{\alpha^{\prime} \beta^{\prime}} \hat{\Lambda}_{M-1, Y}^{\alpha \beta}=\left\langle\alpha^{\prime}, \alpha\left|\hat{\Lambda}_{M, Y^{\prime}} \hat{\Lambda}_{M-1, Y}\right| \beta^{\prime}, \beta\right\rangle= \\
=\left\langle\alpha, \alpha^{\prime}\left|\mathbf{R}(z)^{\dagger} \hat{\Lambda}_{M, Y} \hat{\Lambda}_{M-1, Y^{\prime}} \mathbf{R}(z)\right| \beta, \beta^{\prime}\right\rangle,
\end{array}
$$

where $\mathbf{R}=\mathbf{R}_{n, n^{\prime}}$ and $z=i\left(\nu^{\prime}-\nu\right)$. Any permutation of the separated variables in (19) can be decomposed into elementary steps of type (20), defining a representation of the symmetric group generators

$$
s_{k} \mathbf{Y}=\left(Y_{1}, \ldots, Y_{k+1}, Y_{k}, \ldots, Y_{N}\right) \text {, }
$$

on the space of symmetric spinors

$$
\mathbf{s}_{k}|\boldsymbol{\alpha}\rangle=\mathbf{R}_{n_{k}, n_{k+1}}\left(i \nu_{k+1, k}\right)\left|\alpha_{1}, \ldots, \alpha_{k+1}, \alpha_{k}, \ldots, \alpha_{N}\right\rangle,
$$

and allowing us to state the symmetry of the eigenfunctions under any such permutation,

$$
\Psi^{\alpha \beta}(\mathbf{Y} \mid \mathbf{x})=\Psi^{s_{k}(\boldsymbol{\alpha}, \boldsymbol{\beta})}\left(s_{k} \mathbf{Y} \mid \mathbf{x}\right) .
$$

The scalar product of two eigenfunctions for the model with $N$ sites can be formulated according to (19) in operator form

$$
\left(\hat{\Lambda}_{1, Y_{1}^{\prime}}^{\alpha_{1}^{\prime} \beta_{1}^{\prime}}\right)^{\dagger} \cdots\left(\hat{\Lambda}_{N, Y_{N}^{\prime}}^{\alpha_{N}^{\prime} \beta_{N}^{\prime}}\right)^{\dagger}\left(\hat{\Lambda}_{N, Y_{N}}^{\alpha_{N} \beta_{N}}\right) \cdots\left(\hat{\Lambda}_{2, Y_{2}}^{\alpha_{2} \beta_{2}}\right)\left(\Lambda_{1, Y_{1}}^{\alpha_{1} \beta_{1}}\right),
$$

and its computation can be reduced to that of $N$ scalar products of single-site eigenfunctions, which are straightforwardly given by

$$
\left(\hat{\Lambda}_{1, Y^{\prime}}^{\alpha^{\prime} \beta^{\prime}}\right)^{\dagger} \Lambda_{1, Y}^{\alpha \beta}=\frac{2 \pi^{3}}{n+1} \delta_{n, n^{\prime}} \delta\left(\nu-\nu^{\prime}\right)\left\langle\alpha \mid \alpha^{\prime}\right\rangle^{n}\left\langle\beta \mid \beta^{\prime}\right\rangle^{n} .
$$

The reduction procedure relies on the fact that in the product $\left(\hat{\Lambda}_{M, Y^{\prime}}^{\alpha^{\prime} \beta^{\prime}}\right)^{\dagger}\left(\hat{\Lambda}_{M, Y}^{\alpha \beta}\right)=\left\langle\beta^{\prime}\left|\hat{\Lambda}_{M, Y^{\prime}}^{\dagger}\right| \alpha^{\prime}\right\rangle\left\langle\alpha\left|\hat{\Lambda}_{M, Y}\right| \beta\right\rangle$ the two integral operators can be exchanged decreasing their length by one

$$
\begin{aligned}
& \hat{\Lambda}_{M, Y^{\prime}}^{\dagger} \hat{\Lambda}_{M, Y} \rightarrow \hat{\Lambda}_{M-1, Y} \hat{\Lambda}_{M-1, Y^{\prime}}^{\dagger} \\
& \times \frac{\pi^{4}}{\left[\left(\nu-\nu^{\prime}\right)^{2}+\frac{\left(n-n^{\prime}\right)^{2}}{4}\right]\left[\left(\nu-\nu^{\prime}\right)^{2}+\frac{\left(n+n^{\prime}+2\right)^{2}}{4}\right]},
\end{aligned}
$$

under the assumption $Y \neq Y^{\prime}$, with the additional effect of mixing their auxiliary spinors via the matrix $\mathbf{R}(z)$

$$
\left|\alpha^{\prime}\right\rangle\left\langle\alpha\left|\rightarrow\left\langle\alpha|\mathbf{R}(z)| \alpha^{\prime}\right\rangle, \quad\right| \beta\right\rangle\left\langle\beta^{\prime}\right| \rightarrow\left\langle\beta^{\prime}\left|\mathbf{R}(z)^{\dagger}\right| \beta\right\rangle .
$$


The iterative application of (22) for $M=N, \ldots, 2$, transforms the scalar product of eigenfunctions (19) into the orthogonality condition

$$
\frac{\mu(\mathbf{Y})^{-1}}{N !} \sum_{s \in \mathbb{S}_{N}} \delta\left(\mathbf{Y}-s \mathbf{Y}^{\prime}\right)\left\langle\boldsymbol{\alpha}|\boldsymbol{s}| \boldsymbol{\alpha}^{\prime}\right\rangle\left\langle\boldsymbol{\beta}^{\prime}|\boldsymbol{s}| \boldsymbol{\beta}\right\rangle,
$$

where $\mathbb{S}_{N}$ are the permutations of $N$ objects and we introduced the compact notation

$$
\delta\left(\mathbf{Y}-\mathbf{Y}^{\prime}\right)=\prod_{a=1}^{N} \delta_{n_{a}, n_{a}^{\prime}} \delta\left(\nu_{a}-\nu_{a}^{\prime}\right)
$$

The relations (21), (23) allow us to conjecture the completeness of the proposed eigenfunctions (19) and to define the representation of separated variables as in (8), (9).

Conformal fishnet integrals. - In analogy with the 2D results of [18], employing the results of the previous sections we will compute exactly the four-point correlation function

$$
G_{N, L}=\left\langle\operatorname{Tr}\left[\phi_{1}^{N}\left(x_{1}\right) \phi_{2}^{L}\left(x_{2}\right) \phi_{1}^{\dagger N}\left(x_{3}\right) \phi_{1}^{\dagger L}\left(x_{4}\right)\right]\right\rangle,
$$

for any $N$ and $L$, where $\phi_{1}(x), \phi_{2}(x)$ are the two complex scalar $N_{c} \times N_{c}$ fields which appear in the Lagrangian of the conformal fishnet theory [19] in four dimensions

$$
\mathcal{L}_{\phi}=N_{c} \operatorname{tr}\left[\partial^{\mu} \phi_{1}^{\dagger} \partial_{\mu} \phi_{1}+\partial^{\mu} \phi_{2}^{\dagger} \partial_{\mu} \phi_{2}+(4 \pi)^{2} \xi^{2} \phi_{1}^{\dagger} \phi_{2}^{\dagger} \phi_{1} \phi_{2}\right] .
$$

In the planar limit [31] $N_{c} \rightarrow \infty$ the only connected Feynmann diagram which enters the perturbative expansion in the coupling $\xi^{2}$ of $G_{N, L}$ is given by the integral

$$
\int \frac{d \mathbf{z}}{\left(4 \pi^{2}\right)^{N L}}\left(\prod_{a=0}^{N} \frac{1}{\left(z_{a, b}-z_{a+1, b}\right)^{2}}\right)\left(\prod_{b=0}^{L} \frac{1}{\left(z_{a, b}-z_{a, b+1}\right)^{2}}\right),
$$

where the integration measure is $d \mathbf{z}=\prod_{a, b=1}^{N, L} d^{4} z_{a, b}$ and we set $z_{0 b}=x_{1}, z_{N+1 b}=x_{3}, z_{a 0}=x_{4}, z_{a L+1}=x_{2}$. Such a square-lattice integral can be expressed via the graphbuilding operator (11). Indeed, starting from the fishnet diagram

$$
F_{N, L}=\left(\prod_{a=1}^{N} z_{a a+1}^{2}\right)\left(\mathbb{B}_{N}\right)^{L+1}\left(\prod_{a=1}^{N} \delta^{(4)}\left(z_{a}^{\prime}-z_{a}\right)\right),
$$

one can transform it to (25) by the reductions of external points $z_{a} \rightarrow x_{1}, z_{a}^{\prime} \rightarrow x_{3}$ followed by a conformal transformation. Therefore, as a functions $\mathcal{G}_{N . L}(u, v)$ of the crossratios $u=x_{12}^{2} x_{34}^{2} /\left(x_{13}^{2} x_{24}^{2}\right)$ and $v=x_{14}^{2} x_{23}^{2} /\left(x_{13}^{2} x_{24}^{2}\right)$, the planar limit of (24) is equal to $F_{N, L}$ with reduced external points. According to (6) the integral kernel of $\left(\mathbb{B}_{N}\right)^{L}$ in the space of separated variables is factorized as

$$
\widetilde{\mathbb{B}_{N}^{L}}\left(Y_{1}, \ldots, Y_{N}\right)=\frac{1}{\pi^{2 N L}} \prod_{a=1}^{N}\left[\frac{1}{4 \nu_{a}^{2}+\left(1+n_{a}\right)^{2}}\right]^{L}
$$

In order to restore the $(u, v)$ dependence of $(25)$ one has first to expand the rhs of (26) over the eigenfunctions via the inverse transform (9). Then, by the appropriate reduction of the external points and upon integration of spinors and normalization by the bare correlator, we get

$$
\mathcal{G}_{N, L}(u, v)=\sum_{n \in \mathbb{Z}} \int d \boldsymbol{\nu} \mu(\mathbf{Y}) \prod_{k=1}^{N} \frac{|x|^{-2 i \nu_{k}}(\bar{x} / x)^{\left(n_{k}+1\right) / 2}}{\left(\nu_{k}^{2}+\left(n_{k}+1\right)^{2} / 4\right)^{L+N}},
$$

where $u / v=x \bar{x}, v=1 / \sqrt{(1-x)(1-\bar{x})}$. After the redefinition $n_{k} \rightarrow a_{k}-1, \nu_{k} \rightarrow u_{k}, x \rightarrow z$ it coincides with the result of [20].

We shall conjecture further applications of the separated variables transform (9) to the computation of planar fishnet integrals. An interesting example in this sense is provided by the three-point function of "vacuum" operators

$$
\left\langle\operatorname{Tr}\left(\phi_{1}^{N}\right)\left(x_{1}\right) \operatorname{Tr}\left(\phi_{1}^{L}\right)\left(x_{2}\right) \operatorname{Tr}\left(\phi_{1}^{\dagger N+L}\right)\left(x_{3}\right)\right\rangle .
$$

In the planar limit the perturbative expansion of (28) in the coupling constant consist of regular square lattice diagrams drawn on a three-punctured sphere $S^{2} \backslash\left\{x_{1}, x_{2}, x_{3}\right\}$ as explained in [32] and exemplified in Fig. 4. In the same spirit of "hexagonalization" techniques $[22,23,32,33]$ we perform three cuts on the diagram connecting the punctures, and insert along each cut a sum over the basis (19), labeled by the separated variables

$$
(\mathbf{Y}, \boldsymbol{\alpha}, \boldsymbol{\beta}),(\mathbf{Z}, \lambda, \chi),(\mathbf{U}, \boldsymbol{\kappa}, \boldsymbol{\omega})
$$

where $Y_{a}=\left[\nu_{a}, n_{a}\right], Z_{a}=\left[\mu_{a}, m_{a}\right], U_{a}=\left[\tau_{a}, t_{a}\right]$. Let $M_{i}$ be the number of $\phi_{2} \phi_{2}^{\dagger}$ wrappings around the puncture $x_{i}$ (see Fig. 4). The representation of the two hexagons over the separated variables reads

$$
|H|^{2} \sim|\mathcal{A}|^{2} \prod_{a=1}^{M_{1}+M_{3}}\left[\frac{1}{\nu_{a}^{2}+\frac{\left(n_{a}+1\right)^{2}}{4}}\right]^{N} \prod_{b=1}^{M_{2}+M_{3}}\left[\frac{1}{\mu_{b}^{2}+\frac{\left(m_{b}+1\right)^{2}}{4}}\right]^{L},
$$

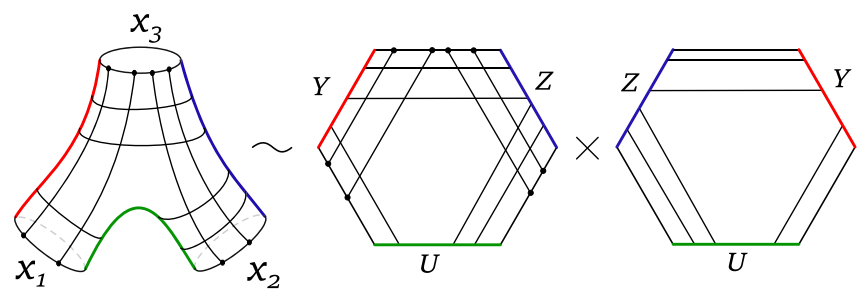

FIG. 4. A Feynmann diagram contributing to the planar limit of $\left\langle\operatorname{Tr}\left(\phi_{1}^{2}\right)\left(x_{1}\right) \operatorname{Tr}\left(\phi_{1}^{2}\right)\left(x_{2}\right) \operatorname{Tr}\left(\phi_{1}^{\dagger 4}\right)\left(x_{3}\right)\right\rangle$ at order $\xi^{28}$ and its decomposition into hexagons. Here $M_{1}=1, M_{2}=2, M_{3}=2$. Each color of a cut corresponds to the insertion of a different set of separated variables, as indicated on the hexagons. 
and the form fa ctor $\mathcal{A}$ is given by the overlapping of three eigenfunctions of type (19) at different values of $x_{0}$

$\mathcal{A}=\int d \mathbf{z} d \mathbf{z}^{\prime} d \mathbf{z}^{\prime \prime} \Psi_{\mathbf{Y}}^{\alpha \beta}\left(\mathbf{z}, \mathbf{z}^{\prime}\right) \Psi_{\mathbf{Z}}^{\lambda \chi}\left(\mathbf{z}, \mathbf{z}^{\prime \prime}\right) \Psi_{\mathbf{U}}^{\kappa \omega}\left(\mathbf{z}^{\prime}, \mathbf{z}^{\prime \prime}\right)$,

for $\quad \mathbf{z}=\left(z_{1}, \ldots, z_{M_{3}}\right), \mathbf{z}^{\prime}=\left(z_{1}^{\prime}, \ldots, z_{M_{1}}^{\prime}\right), \quad$ and $\mathbf{z}^{\prime \prime}=\left(z_{1}^{\prime \prime}, \ldots, z_{M_{2}}^{\prime \prime}\right)$. Finally, the Feynmann integral is recovered by gluing the two hexagons via completeness sums

$$
\sim \sum_{\mathbf{n}, \mathbf{m}, \mathbf{t}} \int d \boldsymbol{\nu} d \boldsymbol{\mu} d \boldsymbol{\tau} \mu(\mathbf{Y}) \mu(\mathbf{Z}) \mu(\mathbf{U}) \int D \alpha \cdots D \boldsymbol{\omega}|H|^{2} .
$$

An interesting reduction of the correlator (28) is obtained setting $L=0$ and degenerating it to the two-point function $\left\langle\operatorname{Tr}\left(\phi_{1}^{N}\right)\left(x_{1}\right) \operatorname{Tr}\left(\phi_{1}^{\dagger N}\right)\left(x_{3}\right)\right\rangle$, for which the planar fishnet lies on a cylinder and it is conformally equivalent to a "wheel" diagram [19,34-36].

As a general fact the diagrams describing the planar limit of (28) develop UV divergences, which in our representation should be contained in the form factor (29). The elaboration of a regularization technique at this level is an intriguing task as it would enable the direct computation of several conformal data in the Fishnet CFT at finite order in the coupling.

Conclusions. - We formulated and solved the spin chain of $S O(1,5)$ conformal spins for any number of sites $N$ and for open boundary conditions, in the principal series representation of zero spin [11]. Its integrability is realized by a commuting family of spectral parameter-dependent operators $\mathbb{Q}_{N}(u)$ that generate the conserved charges of the model. The spectrum of the model is separated into $N$ symmetric contributions, each depending on quantum numbers which for this reason we call separated variables. We explained how to construct the eigenfunctions and prove their orthogonality, extending the logic of [24] to a four-dimensional space-time by means of new integral identities that generalize the star-triangle relation [26] to symmetric traceless tensors.

Our results can be analytically continued from the representation of the principal series to real scaling dimensions, recovering the graph-building operator-introduced in 2D by the authors and V. Kazakov [18] -for the Feynmann diagrams of Fishnet CFT $[19,37]$. The variant of this graph builder with periodic boundary was first introduced in [19] and coincides with the $\hat{B}$ operator of the Fishchain holographic model [38-40]. Following the same steps as [18], we computed the planar limit of the fishnet correlator studied by B. Basso and L. Dixon providing a direct check of the formula (14) of [20].

The separation of variables (SOV) for noncompact spin magnets is a topic which recently attracted great attention [41-46], and SoV features appear in remarkable results of AdS/CFT integrability, for instance [47,48]. It has not escaped our notice that the properties of the proposed eigenfunctions immediately suggest their role in the SOV of the periodic $\mathrm{SO}(1,5)$ spin chain [29], in full analogy with [30]. Moreover it would be interesting to apply our methods to the computation of other classes of Feynmann integrals, for example introducing fermions as in [49,50], or considering any space-time dimension and extending our results to the theory proposed in [51]. In the latter context, the functions (19) for $N=2$ sites have been derived in a somewhat different form and applied to the formulation of the thermodynamic Bethe ansatz equations [52].

Finally we have conjectured how, by means of a cuttingand-gluing procedure inspired by [32], certain planar twoand three-point functions of the fishnet CFT at finite coupling get factorized into simple contributions over the separated variables. This observation puts as a compelling future task the regularization of such formulas, in order to compare the results based on the AdS/CFT correspondence to a direct computation.

We thank B. Basso, A. Manashov, for useful discussions and G. Ferrando and D-1. Zhong for comments on the manuscript. We are grateful to V. Kazakov for participating in the initial stages of the project. The work of S. D. was supported by the RFBR Grant No. 17-01-00283a. The work of E. O. was funded by the German Science Foundation (DFG) under the Research Training Group 1670 and under Germany's Excellence Strategy-EXC 2121 "Quantum Universe"-390833306.

[1] H. Bethe, Z. Phys. 71, 205 (1931).

[2] R. J. Baxter, Exactly Solved Models in Statistical Mechanics (Academic Press, London, 1982).

[3] V. E. Korepin, N. M. Bogoliubov, and A. G. Izergin, Quantum Inverse Scattering Method and Correlation Functions, Vol. 3 (Cambridge University Press, Cambridge, United Kingdom, 1997).

[4] L. D. Faddeev, Symmetries quantiques, in Proc. of Les Houches, Session LXIV 1995 (North Holland, Amsterdam, 1998).

[5] V. S. Fadin, E. Kuraev, and L. Lipatov, Phys. Lett. 60B, 50 (1975).

[6] Y. Y. Balitskii and L. Lipatov, Sov. J. Nucl. Phys. 28 (1978), https://inspirehep.net/literature/137229.

[7] L. N. Lipatov, JETP Lett. 59, 596 (1994); 12, 059 (1994); https://arxiv.org/abs/hep-th/9311037.

[8] N. Beisert et al., Lett. Math. Phys. 99, 3 (2012).

[9] We consider an euclidean space-time in the Letter, without loss of generality respect to the Minkowskian case.

[10] P. Di Francesco, P. Mathieu, and D. Senechal, Conformal Field Theory, Graduate Texts in Contemporary Physics (Springer-Verlag, New York, 1997).

[11] V. K. Dobrev, G. Mack, V. B. Petkova, S. G. Petrova, and I. T. Todorov, Lect. Notes Phys. 63, 1 (1977).

[12] L. N. Lipatov, Phys. Usp. 47, 325 (2004).

[13] E. K. Sklyanin, J. Sov. Math. 47, 2473 (1989).

[14] E. K. Sklyanin, arXiv:hep-th/9211111.

[15] E. K. Sklyanin, Prog. Theor. Phys. Suppl. 118, 35 (1995). 
[16] E. K. Sklyanin, J. Math. Sci. 80, 1861 (1996).

[17] A. B. Zamolodchikov, Phys. Lett. 97B, 63 (1980).

[18] S. Derkachov, V. Kazakov, and E. Olivucci, J. High Energy Phys. 04 (2019) 032.

[19] O. Gürdogan and V. Kazakov, Phys. Rev. Lett. 117, 201602 (2016).

[20] B. Basso and L. J. Dixon, Phys. Rev. Lett. 119, 071601 (2017).

[21] B. Basso, S. Komatsu, and P. Vieira,arXiv:1505.06745.

[22] T. Fleury and S. Komatsu, J. High Energy Phys. 01 (2017) 130.

[23] B. Eden and A. Sfondrini, J. High Energy Phys. 10 (2017) 098.

[24] S. E. Derkachov and A. N. Manashov, J. Phys. A 47, 305204 (2014).

[25] P. P. Kulish, N. Y. Reshetikhin, and E. K. Sklyanin, Lett. Math. Phys. 5, 393 (1981).

[26] M. D’Eramo, G. Parisi, and L. Peliti, Lett. Nuovo Cimento. 2, 878 (1971).

[27] A. P. Isaev, Nucl. Phys. B662, 461 (2003).

[28] A. N. Vasilev, The Field Theoretic Renormalization Group in Critical Behavior Theory and Stochastic Dynamics (Chapman and Hall/CRC, Boca Raton, USA, 2004).

[29] D. Chicherin, S. Derkachov, and A. P. Isaev, J. High Energy Phys. 04 (2013) 020.

[30] S. E. Derkachov, G. P. Korchemsky, and A. N. Manashov, Nucl. Phys. B617, 375 (2001).

[31] G. 't Hooft, Nucl. Phys. B72, 461 (1974).

[32] B. Basso, J. Caetano, and T. Fleury, J. High Energy Phys. 11 (2019) 172.

[33] T. Fleury and S. Komatsu, J. High Energy Phys. 02 (2018) 177.

[34] D. J. Broadhurst, Phys. Lett. 164B, 356 (1985).
[35] E. Panzer, Feynman integrals and hyperlogarithms, Ph.D. thesis, Humboldt U., Berlin, Inst. Math., 2015, arXiv:1506.07243.

[36] N. Gromov, V. Kazakov, G. Korchemsky, S. Negro, and G. Sizov, J. High Energy Phys. 01 (2018) 095.

[37] J. Caetano, O. Gurdogan, and V. Kazakov, J. High Energy Phys. 03 (2018) 077.

[38] N. Gromov and A. Sever, Phys. Rev. Lett. 123, 081602 (2019).

[39] N. Gromov and A. Sever, J. High Energy Phys. 10 (2019) 085.

[40] N. Gromov and A. Sever, J. High Energy Phys. 02 (2020) 035.

[41] J. M. Maillet and G. Niccoli, J. Math. Phys. (N.Y.) 59, 091417 (2018).

[42] J. M. Maillet and G. Niccoli, arXiv:1903.06618.

[43] N. Gromov, F. Levkovich-Maslyuk, and G. Sizov, J. High Energy Phys. 09 (2017) 111.

[44] A. Cavaglià, N. Gromov, and F. Levkovich-Maslyuk, J. High Energy Phys. 09 (2019) 052.

[45] P. Ryan and D. Volin, J. Math. Phys. (N.Y.) 60, 032701 (2019).

[46] N. Gromov, F. Levkovich-Maslyuk, P. Ryan, and D. Volin, Phys. Lett. B 806, 135494 (2020).

[47] I. Kostov, V. B. Petkova, and D. Serban, Phys. Rev. Lett. 122, 231601 (2019).

[48] Y. Jiang, S. Komatsu, and E. Vescovi,arXiv:1906.07733.

[49] V. Kazakov, E. Olivucci, and M. Preti, J. High Energy Phys. 06 (2019) 078.

[50] A. Pittelli and M. Preti, Phys. Lett. B 798, 134971 (2019).

[51] V. Kazakov and E. Olivucci, Phys. Rev. Lett. 121, 131601 (2018).

[52] B. Basso, G. Ferrando, V. Kazakov, and D.-1. Zhong, arXiv:1911.10213. 